$=2 P\left(\frac{3}{2} \cos ^{2} \varphi-\frac{1}{2}\right)$. Here $\varphi$ is the angle between the magnetic interaction axis and the symmetry axis of the electric quadrupole interaction. This gives rise to a distribution of resonance frequencies which has the right order of magnitude when compared to the experimental broadening. In that case the $B_{2}$ and $B_{2} / B_{1}$ values should also be corrected for a shift of the central frequency. A purely magnetic broadening, as well as other explanations, cannot be ruled out. Yet, as we have for the first time the opportunity to study an isolated impurity-vacancy system, it would be very valuable to do more detailed experiments. In this respect it would be highly desirable to repeat these measurements on single crystals: By varying the angle $\varphi$ one could eventually learn more about the origin of the broadening.

The authors with to thank the Belgian Interuniversitair Instituut voor Kernwetenschappen for financial support.

*Aangesteld navorser National Fonds voor Wetenschappelijk Onderzoek.

${ }^{1}$ L. Niesen, J. Lubbers, H. Postma, H. de Waard, and S. A. Drentje, Phys. Lett. B24, 144 (1967).

${ }^{2}$ H. de Waard and S. A. Drentje, Proc. Roy. Soc. Lon- don, Ser. A $\underline{311}, 139$ (1969).

${ }^{3}$ L. C. Feldman and D. E. Murnick, Phys. Rev. B $\underline{5}$, 1 (1972).

${ }^{4}$ H. Pattyn, R. Coussement, G. Dumont, E. Schoeters, R. E. Silverans, and L. Vanneste, Phys. Lett. $\underline{\mathrm{A} 45}$, 131 (1973).

${ }^{5}$ M. Van Rossum, G. Langouche, H. Pattyn, G. Dumont, J. Odeurs, A. Meykens, P. Boolchand, and R. Coussement, J. Phys. (Paris), Colloq. 35, C6-301 (1974).

${ }^{6} \mathrm{H}$. de Waard, R. L. Cohen, S. R. Reintsema, and S. A. Drentje, Phys. Rev. B 10, 3760 (1974).

${ }^{7}$ S. R. Reintsema, S. A. Drentje, P. Schurer, and H. de Waard, Radiat. Eff. 24, 145 (1975).

${ }^{8}$ D. M. Gordon, L. Eytal, H. de Waard, and D. E. Murnick, Phys. Rev. C 12, 628 (1975).

${ }^{9}$ J. Lindhard, M. Scharff, and M. E. Schi $\phi \mathrm{tt}$, K. Dan. Vidensk. Selsk., Mat.-Fys. Medd. 33, 14 (1963).

${ }^{10} \mathrm{~J}$. Odeurs, R. Coussement, G. Dumont, H, Pattyn, E. Schoeters, R. E. Silverans, and L. Vanneste, to be published.

${ }^{11}$ R. E. Silverans, R. Coussement, H. Pattyn, E. Schoeters, and L. Vanneste, Z. Phys. 267, 145 (1974).

${ }^{12}$ R. J. Blin-Stoyle and M. A. Grace, in Handbuch der Physik, edited by S. Flüge (Springer, Berlin, 1957), Vol. 42 , p. 555 .

${ }^{13}$ S. A. Drentje and J. Ekster, J. Appl. Phys. $\underline{45}, 3242$ (1974).

${ }^{14}$ L. S. Kisslinger and R. A. Sorensen, Rev. Mod. Phys. $\underline{35}, 853(1964)$.

\title{
Observation of Surface Excitons in Rare-Gas Solids*
}

\author{
V. Saile, M. Skibowski, $\dagger$ and W. Steinmann \\ Sektion Physik der Universität München, 8000 München 40, Federal Republic of Germany \\ and \\ P. Gürtler \\ II. Physikalische Institut der Universität Hamburg, 2000 Hamburg 50, Federal Republic of Germany \\ and \\ E. E. Koch and A. Kozevnikov \\ Deutsches Elektronen-Synchrotron DESY, 2000 Hamburg 52, Federal Republic of Germany
}

(Received 22 April 1976)

\begin{abstract}
Evidence is obtained for the excitation of surface excitons in solid $\mathrm{Ar}, \mathrm{Kr}$, and $\mathrm{Xe}$ in optical transmission and reflection experiments using synchrotron radiation. They are located at photon energies ranging from $0.6 \mathrm{eV}$ for Ar to $0.1 \mathrm{eV}$ for Xe below the corresponding bulk excitons excited from the valence bands. Their halfwidths $(20-50 \mathrm{MeV})$ are less than half the values found for the bulk excitons. Some are split by an amount considerably smaller than the spin-orbit splitting of the valence bands.
\end{abstract}

In this Letter we report the first evidence for surface excitons in rare-gas solids. They are located at photon energies close to the corresponding bulk valence excitons. We consider them to represent new electronic states which are introduced by the presence of the surface and are spatially confined to a region close to it. They are different from electromagnetic surface 


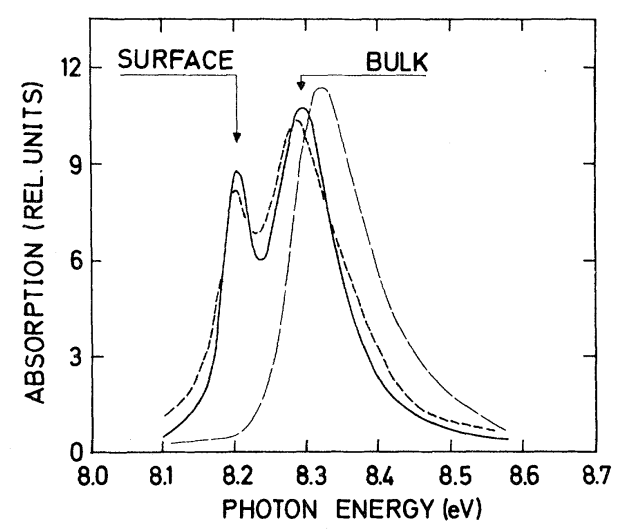

FIG. 1. Absorption of a Xe film of $30 \AA$ thickness in the range of the $n=1$ exciton; the solid curve shows the surface and the bulk exciton, the long-dashed line gives the absorption of the same Xe film with an Ar coating, and the short-dashed curve is the absorption after removal of the Ar overlayer.

waves (surface polaritons), which can be described in the framework of Maxwell's theory by a bulk dielectric constant. By using both absorption and reflection spectroscopy in combination with an electron storage ring as a stable light source for vacuum-uv radiation we have been able to identify surface excitons in addition to the well-known bulk excitons. Although bulk excitons in rare-gas solids have been the subject of considerable theoretical efforts, ${ }^{1-4}$ surface excitons in rare-gas solids have not yet been treated theoretically. Since they represent the lowest excited states in these crystals they may play, however, an important role in the decay of bulk excitons. In particular, processes like photoemission and luminescence from rare-gas solids, where efficient exciton quenching at the surface has been postulated, ${ }^{5,6}$ may be significantly affected by the presence of the observed surface excitons.

Our experiments have been carried out in the synchrotron-radiation laboratory ${ }^{7}$ at the DESY storage ring DORIS. Our apparatus ${ }^{8}$ consists of a high-resolution 3-m normal-incidence monochromator and an ultrahigh-vacuum experimental chamber (base pressure $4 \times 10^{-11}$ Torr) with a Heflow cryostat and a reflectometer. For the reflection measurements thin films of the rare gases were condensed on quartz, KI single crystals, and evaporated $\mathrm{Au}$ subtrates. For the absorption measurements quartz coated with sodium salicylate was used as a substrate. In addition, the transmission of Xe films on a LiF single crystal was measured. The Xe absorption in the range of the $n=1 \Gamma\left(\frac{3}{2}\right)$ valence exciton $(n$ is the principal quantum number) is shown in Fig. 1 for a $30-\AA$-thick film on a $\mathrm{LiF}$ substrate and for the same sample coated with a few monolayers of solid Ar which is transparent in this wavelength region. The absorption spectrum after removal of the Ar layer by gently heating the sample to temperatures above the sublimation temperature of the Ar cover layer is also given. This spectrum is almost identical with that of the original Xe sample. For Ar, the spectra of

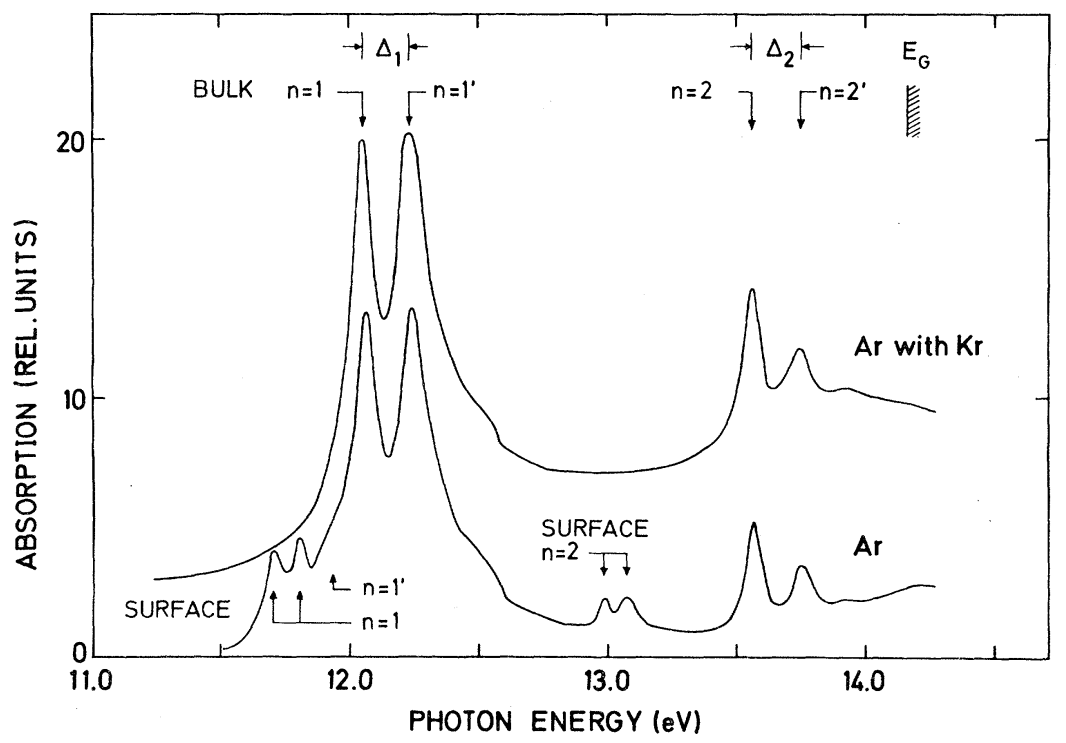

FIG.2. Absorption spectrum of a clean Ar film of $40 \AA$ thickness and of the same Ar film with a Kr overcoating. The surface and bulk excitons are labeled by their main quantum number $n ; \Delta_{1}$ and $\Delta_{2}$ are the splittings resulting from the spin-orbit-split valence bands; $E_{g}$ is the band gap. 
a $40-\AA$-thick film and of the same sample covered with a thin $\mathrm{Kr}$ layer (thickness $\approx 50 \AA$ ) are displayed in Fig. 2 in the range of the valence excitons. In this region $\mathrm{Kr}$ is not completely transparent, but it causes only a structureless background absorption for the $\mathrm{Kr} / \mathrm{Ar}$ sandwich.

On films of Xe, $\mathrm{Kr}$ (no figure), and Ar we observe prominent new structures in addition to the bulk states. Their energies lie slightly below the energies of the well-known absorption bands due to bulk-exciton states. They have considerably smaller half widths than the corresponding bulk excitations. Furthermore a splitting into two $(\operatorname{Kr} n=1, \operatorname{Ar} n=2)$ or even three $\left(\operatorname{Ar} n=1,1^{\prime}\right)$ components is observed. We found the new structures in both the reflection and transmission spectra irrespective of the substrate and independent of the annealing temperature. These results are summarized in Table I. The additional maxima are due to surface excitons. Evidence is based on the following observations:

(1) The surface excitons are only observed under ultrahigh-vacuum conditions. Even in the low $10^{-10}$-Torr range their peak height decreased by more than a factor of 2 within half an hour. A monolayer of adsorbed residual gas made the surface excitons disappear.

TABLE I. Excitation energies $\left(E_{\mathrm{ESS}}\right)$ in $\mathrm{eV}$ and half widths from a rough deconvolution in $\mathrm{MeV}$ (in parentheses) for the excitonic surface states for solid $\mathrm{Ar}, \mathrm{Kr}$, and Xe. Energies for the bulk excitons $E_{\mathrm{BEX}}$ are given for comparison. The radii $r_{n}$ in angstroms have been calculated for the bulk excitons in the Wannier model. $B$ denotes binding energies and $\epsilon_{0}$ the static dielectric constant.

\begin{tabular}{|c|c|c|c|}
\hline & $\mathrm{Ar}$ & $\mathrm{Kr}$ & $\mathrm{Xe}$ \\
\hline \multirow[t]{2}{*}{$E_{\mathrm{E} \mathrm{SS}}, n=1$} & $11.71(50)$ & $9.94(20)$ & $8.21(30)$ \\
\hline & $11.81(50)$ & $10.07(50)$ & \\
\hline \multirow[t]{2}{*}{$n=2$} & $12.99(50)$ & & \\
\hline & $13.07(50)$ & $11.03(50)$ & \\
\hline$n^{\prime}=1$ & $\sim 11.93^{\mathrm{a}}$ & $10.68(50)$ & \\
\hline$E_{\mathrm{BEX}}, n=1$ & 12.06 & 10.17 & 8.28 \\
\hline$n^{\prime}=1$ & 12.23 & 10.86 & 9.51 \\
\hline$n=2$ & 13.57 & 11.23 & 9.07 \\
\hline$n^{\prime}=2$ & 13.75 & & \\
\hline$n=3$ & 13.89 & 11.44 & 9.21 \\
\hline$n=4$ & 13.97 & 11.52 & \\
\hline$B$ & 2.32 & 1.53 & 1.02 \\
\hline$r_{1}$ & 1.9 & 2.5 & 3.2 \\
\hline$r_{2}$ & 7.5 & 9.9 & 12.7 \\
\hline$\epsilon_{0}$ & 1.66 & 1.88 & 2.23 \\
\hline
\end{tabular}

${ }^{a}$ Observable only for films with thickness below $20 \AA$.
(2) The surface excitons disappear upon coating with a different rare-gas film. Instead surface excitons of the cover layer appear.

(3) The dependence of the surface-exciton peak height on the film thickness has been examined from 5 to $100 \AA$. The contribution of the surface excitons to the transmission remains constant whereas the transmission due to the bulk decreases with increasing film thickness. From the thickness dependence we estimate that the absorption due to surface excitons is confined to one or two layers at the sample-vacuum boundary. For thick films ( $\geqslant 100 \AA$ ) the surface excitons show up only as small humps at the low-energy side of the bulk exciton bands.

(4) The Xe surface-state structure reappears after the Ar cover layer is removed.

A detailed interpretation of the experimental results has to account for the position of the surface excitons, their splitting, and their oscillator strength. A general approach may start from electronic surface bands with an appropriate gap energy and a symmetry lower than the bulk one eventually lifting bulk degeneracies. Since nothing is known yet about electronic surface bands on rare-gas solids this cannot be further substantiated. When directly compared to bulk excitations the surface excitations can be viewed as caused by changes in the spatial environment while approaching the surface. ${ }^{9}$ This will give rise to a reduction of dielectric screening, changes in the orthogonality corrections, altered boundary conditions for the electronic wave function, and an additional surface field with a symmetry different from that in the bulk. A comprehensive theory would have to take into account these changes in total.

In order to illuminate partial aspects we consider first the possible effects of reduced dielectric screening in the region close to the surface. Using the same theoretical model for surface excitons as for bulk excitons, namely the effectivemass approximation which predicts an exciton binding energy $B=\mu \epsilon_{\mathrm{eff}}^{-2} B_{H}\left(B_{H}=1 \mathrm{Ry}, \mu\right.$ is the effective reduced electron mass in free-electron units, and $\epsilon_{\text {eff }}$ is an average dielectric constant), one would expect a larger binding energy for a decreasing dielectric constant and therefore a lowered excitation energy as observed, provided the effective mass and the gap energy do not change significantly. This approximation may be applicable in the case of the large-radius excitons as for the $n=2$ exciton in $\mathrm{Kr}, r_{n=2}(\mathrm{bulk})=9.9 \AA$. If in a crude approximation one as sumes $\epsilon_{\text {eff }}=\frac{1}{2}(1$ 
$\left.+\epsilon_{\text {bulk }}\right)$, close to the surface the binding energy is increased by $0.27 \mathrm{eV}$ which agrees with the experimental value $0.2 \mathrm{eV}$. For the $n=2$ exciton in Ar with a bulk radius of $7.5 \AA$ the calculated increase in binding energy of $0.3 \mathrm{eV}$ compares with an observed value of 0.5 to $0.6 \mathrm{eV}$.

For the $n=1$ bulk excitons the radii are comparable to or smaller than the nearest-neighbor distance (see $r_{n=1}$, Table I). In this case the real potential will no longer vary as $1 / r(r$ is the electron-hole separation) with a uniform dielectric constant. Within the pseudopotential approach three different correction factors have been considered in order to calculate the energies in the bulk: a spatially varying $\epsilon$, the actual band structure instead of parabolic bands, and the orthogonality of the wave function to core states. ${ }^{2}$ For solid $\mathrm{Ar}$ and $\mathrm{Kr}$ the net result of these three corrections, the central-cell correction, leads to a blue shift of the $n=1$ bulk exciton energies. Similar corrections might also be important for the surface excitons.

A quantitative change of the factors determining the excitation energy in the surface region remains to be calculated. A recent paper by Andreoni, Altarelli, and Bassani ${ }^{4}$ points in the right direction. These authors demonstrated that for the bulk $n=1$ exciton of Ar the electron and the hole are confined to the same unit cell; i.e. the spherical part of the Wannier function is more strongly localized than the $4 s$ atomic wave function of Ar. A surface would lower this restriction and would yield excitation energies closer to the atomic values, presumably not far from those observed for the surface excitons in our experiments.

In addition to the excitation energies the splitting observed for the surface excitons in Ar and $\mathrm{Kr}$ has to be considered. Spin-orbit splitting can be rejected since the observed values for the splitting, $0.10 \mathrm{eV}(n=1), 0.08 \mathrm{eV}(n=2)$ for Ar, and $0.13 \mathrm{eV}(n=1)$ for $\mathrm{Kr}$, are too small compared to the spin-orbit splitting of the valenceband states at the $\Gamma$ point which are $0.18 \mathrm{eV}(0.22)$ and $0.64 \mathrm{eV}(0.67)$ for $\mathrm{Ar}$ and $\mathrm{Kr}$, respectively (the values in parentheses are the spin-orbit splittings in the gas phase). ${ }^{10}$ Surface-field splitting, as recently discussed for $\mathrm{MgO},{ }^{11}$ would provide an explanation. In the bulk the upper valence bands $\left(j=\frac{3}{2}\right)$ are degenerate at $\Gamma$. This degeneracy can be lifted in the surface layer with lower symme- try than the cubic crystal symmetry. Hence one could expect two separated excitonic surface transitions from the upper $j=\frac{3}{2}$ valence-band split by the surface field.

The data which we have presented above provide the possibility to test theoretical models for surface excitons in simple insulators. ${ }^{12}$ New information on the bulk excitons, concerning excitation energies, oscillator strengths, exchange interaction, and line shapes, was also obtained in our experiments. These results are the subject of a forthcoming paper. ${ }^{13}$

\footnotetext{
*Work supported by Deutsche Forschungsgemeinschaft DFG and Bundesministerium für Forschung und Technologie BMFT.

$\dagger$ Now at Universität Kiel, $2300 \mathrm{Kiel}$, Germany.

$\$$ Permanent address: Technical University Tomsk, Tomsk, U.S. S. R.

${ }^{1}$ See, for instance, R. S. Knox, Theory of Excitons (Academic, New York, 1963).

${ }^{2} \mathrm{~J}$. Hermanson and J。 C. Phillips, Phys. Rev. 150, 652 (1966); J. Hermanson, Phys. Rev. 150, $660 \overline{(1966) .}$

${ }^{3}$ U. Rössler and O. Schütz, Phys。 Status Solidi (b) $\underline{56}$, 483 (1973).

${ }^{4}$ W. Andreoni, M。 Altarelli, and F. Bassani, Phys。 Rev. B 11, 2352 (1975), and references therein。

${ }^{5}$ Z. Ophir, B。 Raz, J. Jortner, V. Saile, N. Schwentner, E. E. Koch, M. Skibowski, and W. Steinmann, J. Chem. Phys. 62, 650 (1975); D. Pudewill, F.-J. Himpsel, V. Saile, N. Schwentner, M. Skibowski, E. E. Koch, and J. Jortner, to be published.

${ }^{6}$ Ch. Ackermann, R. Brodmann, U. Hahn, A. Suzuki, and $\mathrm{G}_{0}$ Zimmerer, to be published.

${ }^{7}$ E. E. Koch, C.Kunz, and E. W. Weiner, to be published.

${ }^{8}$ V. Saile, P. Gürtler, E. E. Koch, A. Kozevnikov, M. Skibowski, and W. Steinmann, to be published。

${ }^{9}$ M. R. Philpott, J。Chem. Phys. 60 , 1410 (1974);

V. I. Sugakov, Ukr. Fiz. Zh. 14, 1425 (1969) (Ukr. Phys. J. 15, 2060 (1970)].

${ }^{10}$ B. Sonntag, in Rare Gas Solids, edited by M. L. Klein and J. A。Venables (Academic Press, New York, 1976).

${ }^{11}$ V. E. Henrich, G. Dresselhaus, and H. J. Zeiger, Phys. Rev. Lett. 36, 158 (1976).

${ }^{12}$ G. J. Lapeyre and J. Anderson [Phys. Rev. Lett. 35, 117 (1975)] have obtained evidence for surface excitons in photoemission from GaAs (110) faces. They interpret their data as a core surface exciton associated with the Ga dangling bonds.

${ }^{13} \mathrm{~V}$. Saile et al., to be published.
} 